\title{
Biodiversity of the Indonesian marine sponge genus Aaptos with molecular networking approach
}

\author{
Viqqi Kurnianda ${ }^{1}$, Ulung Jantama Wisha ${ }^{2}$, and Musri Musman ${ }^{3 *}$ \\ ${ }^{1}$ Department of Marine Science, Faculty of Marine and Fisheries, Syiah Kuala University, Banda Aceh 23111, Indonesia \\ ${ }^{2}$ Research Institute for Coastal Resources and Vulnerability, Ministry of Marine Affairs and Fisheries, Padang 25245, Indonesia \\ ${ }^{3}$ Department of Chemistry Education, Faculty of Teacher Training and Education, Syiah Kuala University, Banda Aceh 23111, \\ Indonesia
}

\begin{abstract}
Many natural products have been isolated from the cryptic species of the Indonesian marine sponge. Despite this, it remains unclear how sponges produce such a diverse array of metabolites and their relationship to sponge diversity. During our expedition in 2017-2019, we collected the Indonesian marine sponge of the genus Aaptos, which was found surrounding Weh and Aceh Islands, the northwesternmost part of the Indonesian archipelago. All specimens were subjected to a feature-based molecular networking approach to understand their relationship. The molecular phylogenetic clades of genus Aaptos were examined by cytochrome oxide I (COI) while the presence of their chemotypes were confirmed by chemical analysis based on bioassay-guided separation with several steps of chromatography. As results, the presence Aaptos suberitoides, Aaptos aaptos, Aaptos nigra, Aaptos lobata, and Aaptos sp. were confirmed by the molecular phylogenetic. On the other hand, chemical analysis showed the presence of alkaloid-class molecules on all specimens. Interestingly, feature-based molecular networking revealed the whole figure of cryptic species were formed a clear and distinct group together with their chemotypes. Consequently, it confirmed that the relationship between the presence of each species as well as its chemotype can be distinguished clearly. In conclusion, we suspect the chemical analysis of the genus Aaptos have unknowingly conflated between different cryptic species, resulting in the seemingly idiosyncratic chemical variations.
\end{abstract}

\section{Introduction}

Metabolites found in marine ecosystems exhibit wide structural diversity and bioactive properties [1]. Sponge, which belongs to the phylum Porifera, is known to contain a variety of secondary metabolites [2]. Interestingly, compounds from sponges have often been found to be promising pharmaceutical agents [3]. To date, FDA has been approved 3 marine drugs derived from sponges such as anticancer cytarabine (ara-C) and anticancer Eribulin mesylate, and antiviral vidarabine (ara-A) [4-7].

Sponge genus Aaptos is found in a wide range of ecosystems, including the South China Sea, Japanese, Indonesian, and Caribbean waters [8-11]. The first discovery was conducted by Nakamura and co-workers from an Okinawan sponge Aaptos aaptos which contain aaptamine, an alkaloidal molecule, in 1981 [12]. To date, many other research groups throughout the world have also carried out chemical analyses on different Aaptos species to investigate the structural diversity [1315].

During our expedition around Weh and Aceh Islands in the early 2017, we discovered the marine sponge of the genus Aaptos. Since there are no reported papers on the Aaptos sponges around that area to date, we examined both genetic and chemical contents of them. In this paper, we revealed the whole figure of genetic and chemical diversity on Aaptos sponges around Weh and Aceh Islands using the metabolomics approach.

\section{Materials and methods}

\subsection{Materials}

Nuclear Magnetic Resonance (NMR) $500 \mathrm{MHz}$ spectroscopy (Bruker Biospin, Billerica, MA, USA) in $\mathrm{CDCl}_{3}$ (Cambridge Isotope Labs). All extracts were injected into LC-MS/MS data using a reversed-phase analytical HPLC column $(5 \mu \mathrm{m}, 250 \mathrm{~mm} \times 4.6 \mathrm{~mm})$. Peaks were detected by positive ion mode of highresolution MS/MS system.

\subsection{Extraction and isolation}

Specimens were collected by hand using SCUBA at 10 to $30 \mathrm{~m}$ depth off surrounding Weh and Aceh Islands from 2017 to 2019 (Fig. 1). The specimens were kept in the freezer during the trip. Furthermore, all specimens consist of 209 specimens from Weh and 347 specimens from Aceh Islands were extracted by acetone. After partitioning and concentrating the extracts, the organic layer was used for purification and metabolomics purposes. On the other hand, all extracts which contain

* Corresponding author: musrimusman@unsyiah.ac.id 
alkaloidal markers were purified to obtain pure material used for the chemical standard.

\subsection{Phylogenetic analysis}

All crude extracts $(10.0 \mathrm{mg})$ were extracted using Qiagens' DNeasy Blood \& Tissue Kit (Qiagen, Hilden, Germany), followed by PCR amplification using dmaW forward (5'-TTGATCTGGAGTGGTTCCGC-3') and reverse (5'-CGTTCATGCCGAAGGTTGTG-3') under the standard condition. Amplicons were analysed by AB3130 with ABI PRISM BigDye Terminator Cycle Sequence Kit v1.1, and sequence alignment used MEGA v5.1.

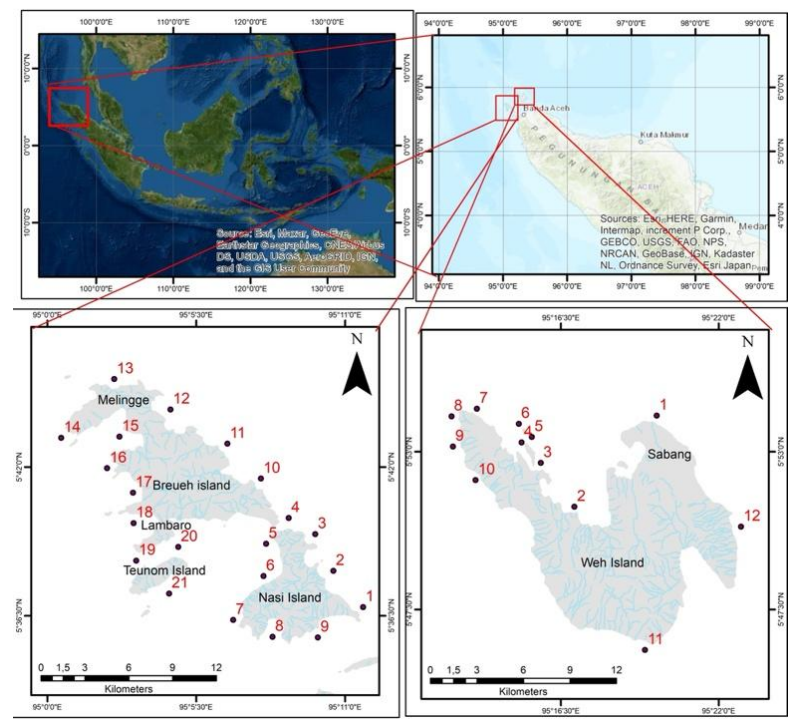

Fig. 1. The collection sites.

\subsection{Metabolomics approach}

The presence of the marker alkaloidal was confirmed by a 'H NMR spectra comparison. On the other hand, For LC-MS/MS analysis, MeOH solution (20 $\mu \mathrm{L})$ containing each crude extract was prepared. We applied a flow rate of $0.5 \mathrm{~mL} / \mathrm{min}$ under positive ion mode of high resolution to samples ranging from 50 to $1000 \mathrm{~m} / \mathrm{z}$. Furthermore, all raw data were converted to mzXML format, and it was uploaded online with reference number MSV000088027 (https://massive.ucsd.edu) [16]. To further analysis, we subjected them to MZmine2 software. On the GNPS website (http://gnps.ucsd.edu), quantitative and MS/MS tables have been uploaded for use in a feature-based molecular networking study [17]. A cosine score of 0.6 was applied to the parameters with a maximum of 10 matched peaks. Then, by using Cytoscape 3.7.2 version, the results were visualized [18].

\subsection{Spatial analysis of the Sponge genus Aaptos}

The specimen data collected at each observation station were then spatially analysed using an interpolation method. Inverse Distance Weighted (IDW) interpolation was employed to perform the distribution model of the detected individual. This method corresponds to the
Indonesian Standard (SNI 7644:2010) for oceanographic-spatial data provider. IDW method can calculate faster than the other methods such as kriging, even though the calculation quality is categorised to "acceptable" [19]. IDW assumes the value resulted from interpolation process as similar as the closer sampled data compared to the distant ones. The distance among the interpolated points toward the reference is going to influence the weight of calculation [20]. The interpolation results were limited to the initial data so that the product will not be exceeded or extrapolated.

\section{Results and discussion}

All specimens both from Weh and Aceh Islands were examined by 'H NMR comparison with those reported [21-25]. As results, all extracts were identified as known compounds. However, since there was a methoxy moiety was introduced with those reported, it was likely a typical solvolysis product during chemical works. Careful analysis by LC-MS/MS revealed the presence of phenolic groups on all extracts (Fig. 2). It also confirmed that those likely reported to be artifacts by solvolysis reaction to yield methoxy derivatives [26].

Since there is quite similar morphology on the marine sponge of the genus Aaptos, we examined the whole diversity figure using molecular networking approach. After obtaining the MS/MS data, all raw data were subjected to MZmine2 for feature detection, grouping, and alignment. By uploading the results on the GNPS web platform, the feature-based on molecular networking may reveal the whole figure of diversity in the sponge of the genus Aaptos relating to their chemotypes. As results, the presence of some clades on the sponge of the genus Aaptos were confirmed by the phylogenetic tree. Moreover, feature-based on molecular networking revealed the whole figure of their diversity (Fig. 3).

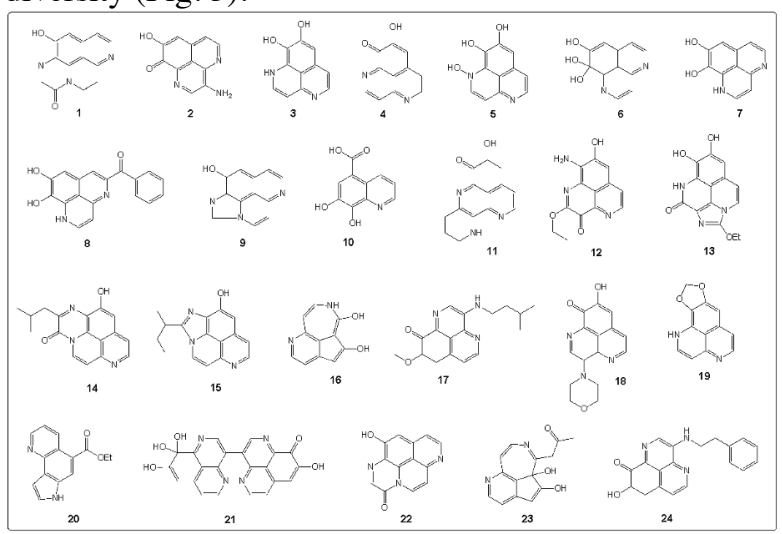

Fig. 2. Chemotypes of sponge of the genus Aaptos around Weh and Aceh islands.

To understand how the sponge was spread, we used the distribution analysis to examine their spread (Figs. 4-5). As results, A. aaptos, A. lobata, A. nigra, and A. suberitoides were spread in the northern part while Aaptos sp. was spread in the western part of Weh Island (Fig. 4). The characteristic of geography in Aceh Island may introduce unique sponge spread in the area. The presence of $A$. nigra, and $A$. suberitoides were discovered in the northern part of the collection site in 
Nasi Island, meanwhile, Aaptos sp. was found in the southern part of Nasi Island. On the other hand, the sponge of the genus $A$. lobata was spread in both northern and southern parts of Breueh Island (Fig. 5). We suspected that $A$. lobata is a more tolerant sponge of big waves and strong currents from the Indian Ocean in the western part of Aceh Island. As the sponges contain the potential of secondary metabolite, it also gives us an opportunity to discover the resources for the pharmaceutical sciences purposes.

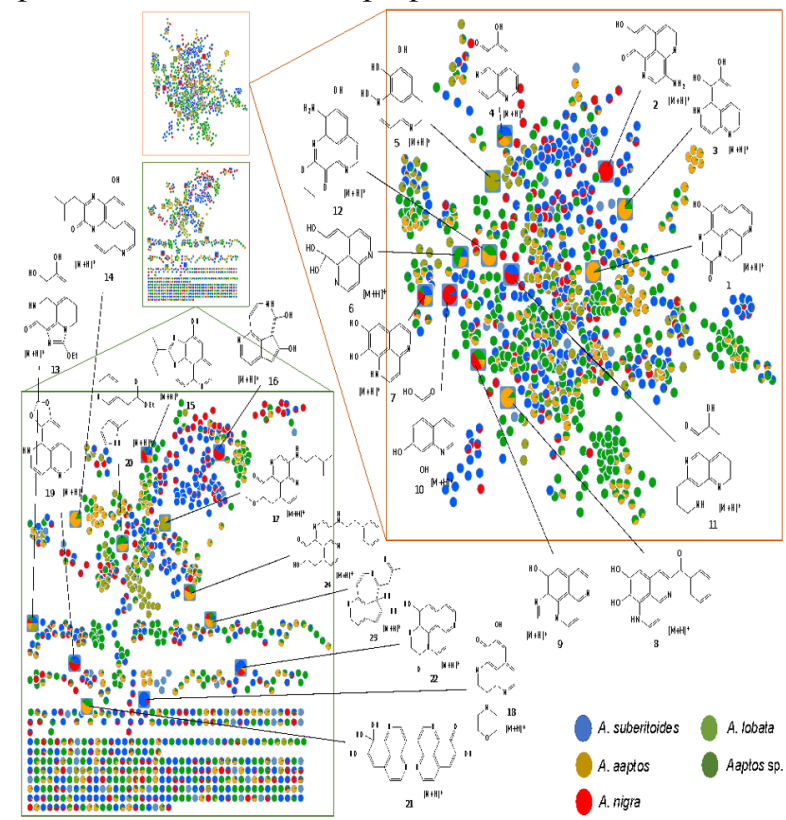

Fig. 3. Feature-based on molecular networking.

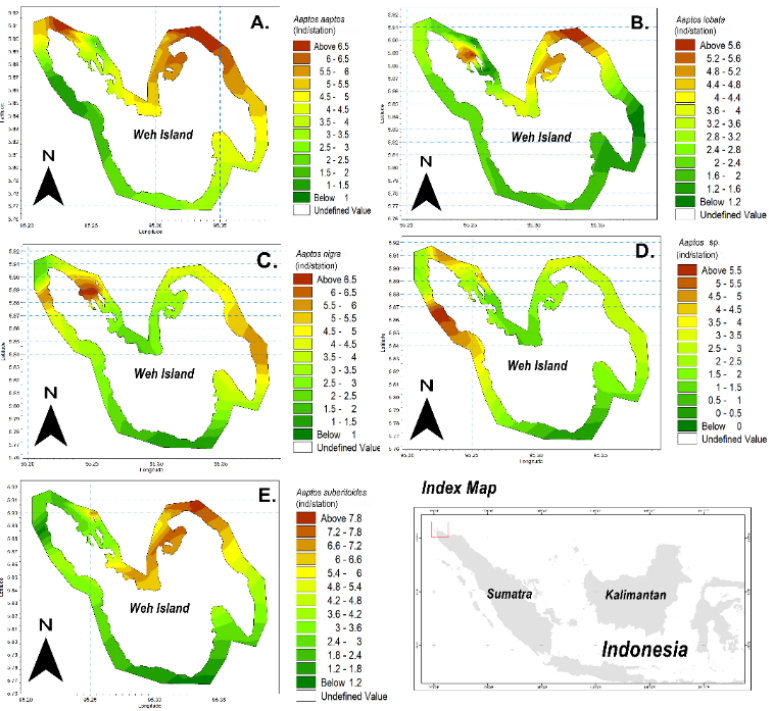

Fig. 4. Distribution of the genus Aaptos in Weh Island; (a) Aaptos aaptos, (b) Aaptos lobata, (c) Aaptos nigra, (d) Aaptos sp. and (e) Aaptos suberitoides.

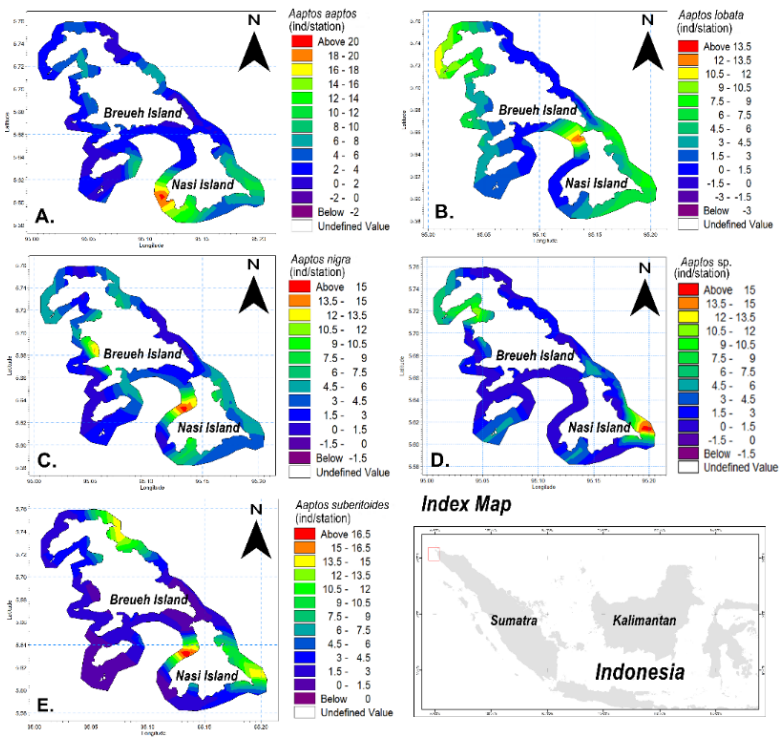

Fig. 5. Distribution of the genus Aaptos in Aceh Island; (a) Aaptos aaptos, (b) Aaptos lobata, (c) Aaptos nigra, (d) Aaptos sp., and (e) Aaptos suberitoides.

\section{Conclusion}

The presence of Aaptos suberitoides, Aaptos aaptos, Aaptos nigra, Aaptos lobata, and Aaptos sp. from the Indonesian marine sponge of the genus Aaptos were confirmed by the molecular phylogenetic. The chemical analysis showed the presence of alkaloid-class molecules on all specimens. Interestingly, feature-based molecular networking revealed the whole figure of cryptic species were formed a clear and distinct group together with their chemotypes. Further analysis revealed that, A. aaptos, A. lobata, A. nigra, and $A$. suberitoides were spread in the northern part while Aaptos sp. was spread in the western part of Weh Island. Moreover, the presence of A. nigra, and A. suberitoides were discovered in the northern part of the collection site in Nasi Island, meanwhile, Aaptos sp. was found in the southern part of Nasi Island. On the other hand, the sponge of the genus $A$. lobata was spread in both northern and southern parts of Breueh Island. As the sponges contain the potential of secondary metabolite, it also gives us an opportunity to discover the resources for the pharmaceutical sciences purposes.

\section{References}

1. Nadar, V.M. S. Manivannan., R. Chinnaiyan., M. Govarthanan., and Ponnuchamy, K. Chem. Biol. Drug Des. 2021 (in press).

2. He, Q., S. Miao., N. Ni., Y. Man., K. Gong. Nat. Prod. Commun. 2020, 15, 1934578 X20951439.

3. Pham, C.D., R. Hartmann., W.E. Müller., N.J. de Voogd., D. Lai,. P.J. Proksch. J. Nat. Prod. 2013, 76, 103-106.

4. Calcabrini, C., E. Catanzaro., A. Bishayee., E. Turrini., C. Fimognari. Mar. drugs 2017, 15, 310.

5. Sagar, S., M. Kaur., K.P. Minneman. Mar drugs 2010, 8, 2619-2638. 
6. Gyawali, B., A.S. Kesselheim. Nat. Rev. Clin. Oncol. 2021, 1-2.

7. Del Paggio, J.C., I.F, Tannock. Lancet Oncol. 2019, 20, 1065-1069.

8. Elissawy, A.M., D.E. Soleiman., N. Mehdinezhad., M.L. Ashour., P.P. Mohammadi. Biomolecules 2021. 11, 258.

9. Trang, D.T., B.H. Tai., D.T.T. Hang., P.H. Yen., N.X. Nhiem., P.V. Kiem. Nat. Prod. Res. 2021, 110.

10. Fristiohady, A., B. Sadarun., W. Wahyuni., M.H. Malaka., F. Ahmad., F. Malik., L.O.M.J. Purnama., I. Sahidin. J Appl Pharm Sci. 2020, 10, 081-089.

11. Anjum, K., S.Q. Abbas., S.A.A. Shah., N. Akhter., S. Batool., S.S. ul Hassan. Biomol. Ther. 2016, 24, 347.

12. Nakamura, H., J.I. Kobayashi., Y. Ohizumi., Y. Hirata. Tetrahedron Lett. 1982, 23, 5555-5558.

13. Nguyen, T.L.A., T.H.N. Doan., D.H. Truong., N.T.A. Nhung., D.T. Quang., D. Khiri., S. Taamalli., F. Louis., A. El Bakali., and D.Q. Dao. RSC Adv. 2021, 11, 21433-21446.

14. Paliwal, D., S. Srivastava., P.K. Sharma., I. Ahmad. Curr. Tradit. Med. 2021, 7, 93-106.

15. Chabowska, G., E. Barg., A. Wójcicka. Molecules 2021, 26, 4324.

16. MassIVE 2021. https://massive.ucsd.edu/. Accessed on August 27, 2021

17. Nothias, L.F., D. Petras., R. Schmid., K. Dührkop., J. Rainer., A. Sarvepalli., I. Protsyuk., M. Ernst., H. Tsugawa., M. Fleischauer., F. Aicheler. Nat. Methods 2020, 17, 905-908.

18. Aron, A.T., E.C. Gentry., K.L. McPhail., L.F. Nothias., M. Nothias-Esposito., A. Bouslimani., D. Petras., J.M. Gauglitz., N. Sikora., F. Vargas., J.J. van Der Hooft. Nat. protocols 2020, 15, 19541991.

19. Qin, R., L. Lin. Computers and Géosciences 2017, 103, 70-79.

20. Childs, C. Interpolating Surface in ArcGIS Spatial Analysis. ESRI Education Services 2004.

21. Jang, K.H., S.C. Chung., J. Shin., S.H. Lee., T.I. Kim., H.S. Lee., K.B. Oh. Bioor. Med. Chem. Lett. 2007, 17, 5366-5369.

22. Pham, C.D., R. Hartmann., W.E. Müller., N.J. de Voogd., D. Lai,. P.J. Proksch. J. Nat. Prod. 2013, 76, 103-106.

23. Yu, H.B., F. Yang., F. Sun., G.Y. Ma., J.H. Gan., W.Z. Hu., B.N. Han., W.H. Jiao., H.W. Lin. J. Nat. Prod. 2014, 77, 2124-2129.

24. Wu, C.F., M.G. Lee., M. El-Shazly., K.H. Lai., S.C. Ke., C.W. Su., S.P. Shih., P.J. Sung., M.C. Hong., Z.H. Wen., M.C. Lu. Mar. Drugs 2018, 16, 18.

25. He, Q., S. Miao., N. Ni., Y. Man., K. Gong. Nat. Prod. Commun. 2020, 15, $1934578 X 20951439$.

26. Kurnianda, V., H. Fujimura., Y. Kanna., J. Tanaka. Chem. Lett. 2021, 50, 220-222. 